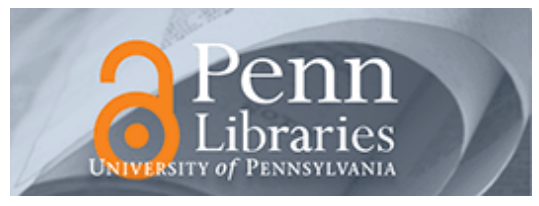

University of Pennsylvania

ScholarlyCommons

Finance Papers

Wharton Faculty Research

6-1998

\title{
The Mix and Scale of Factors with Irreversibility and Fixed Costs of Investment
}

Andrew B. Abel

University of Pennsylvania

Janice C. Eberly

Follow this and additional works at: https://repository.upenn.edu/fnce_papers

Part of the Finance and Financial Management Commons, and the Social and Behavioral Sciences Commons

\section{Recommended Citation}

Abel, A. B., \& Eberly, J. C. (1998). The Mix and Scale of Factors with Irreversibility and Fixed Costs of Investment. Carnegie-Rochester Conference Series on Public Policy, 48 101-135. http://dx.doi.org/ 10.1016/S0167-2231(98)00018-9

This paper is posted at ScholarlyCommons. https://repository.upenn.edu/fnce_papers/49

For more information, please contact repository@pobox.upenn.edu. 


\title{
The Mix and Scale of Factors with Irreversibility and Fixed Costs of Investment
}

\author{
Abstract \\ When factors of production can be adjusted costlessly, the mix of factors can be considered separately \\ from their scale. We examine factor choice and utilization when investment is irreversible and subject to a \\ fixed cost, so that the capital stock is a quasi-fixed factor that is adjusted infrequently and by discrete \\ amounts. We derive and analyze analytic approximations for optimal investment behavior, and show how \\ the quasi-fixity of capital eliminates the dichotomy between factor mix and scale. We show that the quasi- \\ fixity of capital can give rise to labor hoarding, even when labor is a purely flexible factor.

\section{Disciplines} \\ Finance and Financial Management | Social and Behavioral Sciences
}


NBER WORKING PAPER SERIES

THE MIX AND SCALE OF FACTORS

WITH IRREVERSIBILITY AND FIXED

COSTS OF INVESTMENT

Andrew B. Abel

Janice C. Eberly

Working Paper 6148

http://www.nber.org/papers/w6148

NATIONAL BUREAU OF ECONOMIC RESEARCH

1050 Massachusetts Avenue

Cambridge, MA 02138

August 1997

We thank Susanto Basu, Marcelo Veracierto, the Penn Macro Lunch Group, and seminar participants at the Federal Reserve Banks of Minneapolis, New York, and St. Louis, and the Board of Governors of the Federal Reserve System for helpful comments. This paper is part of NBER's research program in Economic Fluctuations and Growth. Any opinions expressed are those of the authors and not those of the National Bureau of Economic Research.

(C) 1997 by Andrew B. Abel and Janice C. Eberly. All rights reserved. Short sections of text, not to exceed two paragraphs, may be quoted without explicit permission provided that full credit, including $(\mathbb{C}$ notice, is given to the source. 
The Mix and Scale of Factors with

Irreversibility and Fixed Costs of Investment

Andrew B. Abel and Janice C. Eberly

NBER Working Paper No. 6148

August 1997

JEL No. E22

Economic Fluctuations and Growth

\begin{abstract}
When factors of production can be adjusted costlessly, the mix of factors can be considered separately from their scale. We examine factor choice and utilization when investment is irreversible and subject to a fixed cost, so that the capital stock is a quasi-fixed factor that is adjusted infrequently and by discrete amounts. We derive and analyze analytic approximations for optimal investment behavior, and show how the quasi-fixity of capital eliminates the dichotomy between factor mix and scale. We show that the quasi-fixity of capital can give rise to labor hoarding, even when labor is a purely flexible factor.
\end{abstract}

Andrew B. Abel

Department of Finance

The Wharton School

University of Pennsylvania

3620 Locust Walk

Philadelphia, PA 19104-6367

and NBER

abel@wharton.upenn.edu
Janice C. Eberly

Department of Finance

The Wharton School

University of Pennsylvania

3620 Locust Walk

Philadelphia, PA 19104-6367

and NBER

eberlyj@wharton.upenn.edu 


\section{Introduction}

A firm's production decision can be viewed as a two-stage problem. First, conditional on a level of output, cost minimization determines the optimal mix of factors of production and the implied cost of production. Second, the demand curve, together with the cost function obtained from cost minimization, is used to determine the profitmaximizing level of output, or the scale of operation. Because the optimal mix of factors is conditional on the scale of operation, the choice of scale and mix are related in general. However, when all factors of production can be costlessly adjusted and the production function is homothetic, these two choices - mix and scale - are independent choices. The mix of factors (say, the capital-labor ratio) depends only on relative rental prices,' and the scale of operations is determined by the levels of productivity and demand. We introduce irreversibility and fixed costs of adjusting capital, and show that the resulting quasi-fixity of capital eliminates the dichotomy between mix and scale. The quasi-fixity of any factor directly affects the scale of operations by preventing short-run adjustments in capacity. Moreover, the use of other factors is affected. In general, the mix of factors depends on the scale of operations, and even when it does not, the link between factors generates dynamic behavior for the flexible factors mimicking that of the quasi-fixed factor.

We develop a model in which a firm faces two costs of adjusting its capital stock: it incurs a fixed cost every time it acquires capital, and it cannot sell its capital at a positive price, so that capital investment is effectively irreversible. ${ }^{2}$ As emphasized in single-factor models, such as Caballero and Leahy (1996), fixed costs of adjusting the capital stock have important implications for the dynamic behavior of optimal factor usage. Of course, single-factor models focus exclusively on the scale of the factor since 
they obviously cannot be used to analyze the mix of factors. ${ }^{3}$ Investment takes place infrequently at points of time that are optimally chosen by the firm as a function of underlying (stochastic) state variables. During the period of time between two consecutive instants of investment, the firm does not purchase any capital, so that (except for depreciation) the capital stock is fixed. These intervals of time without capital investment correspond to the Marshallian notion of the short run. We model the short run as the consequence of optimal intertemporal behavior so that the length of the short run is endogenous and stochastic. We calculate the expected length of the short run and show how it depends on the environment of the firm.

During the short run, the firm chooses the amounts of costlessly adjustable factors and chooses the level of factor utilization. For simplicity, we restrict attention to a single costlessly adjustable factor of production, and we interpret this factor as labor. With this interpretation, our model generates employment behavior consistent with labor hoarding. Specifically, in response to a decline in the demand for its product, a firm may reduce its output by reducing its utilization of labor and capital, while holding [replace "the capitallabor ratio" by: both the capital stock and employment] unchanged. Since the capitallabor ratio only depends on relative rental prices in this case, the dichotomy between mix and scale of factor use is restored; output falls as a result of the drop in utilization while the capital stock and employment remain unchanged. The drop in output without any change in employment is an extreme example of labor hoarding. Interestingly, this laborhoarding behavior arises even though labor is costlessly adjustable.

When this extreme form of labor hoarding is not in force, the mix and scale of factors are related. In such a case, a firm may respond to a drop in demand by reducing 
its utilization of factors and by reducing its employment of labor, but the percentage reduction in labor may be smaller than the percentage reduction in output. An observer who ignores the reduction in factor utilization and ascribes the reduction in output entirely to a reduction in labor would estimate a short-run elasticity of output with respect to labor that exceeds one-finding short-run increasing returns to labor. Although this link between unobserved utilization and short-run increasing returns to labor has been noted in the literature (for example, Burnside, Eichenbaum, and Rebelo (1993)), our analysis is the first to explore this link in the context of a fully intertemporal model of optimal behavior that does not impose employment rigidities and allows for endogeneity of the short run.

In our framework, the optimal investment behavior of a firm is easily characterized in terms of a state variable $y$ that is monotonically related to the marginal revenue product of capital. This state variable summarizes the stochastic behavior of productivity, demand, and the operating costs of capital and labor. In the short run, the state variable $y$ evolves stochastically, rising when investment becomes more attractive, and falling when investment becomes less attractive. As long as the state variable $y$ remains within an optimally-determined range of inaction, the firm does not purchase capital. However, if $y$ grows and reaches an optimal trigger value, the firm pays the fixed cost and buys a discrete amount of capital. This discrete increase in the capital stock causes the marginal revenue product of capital to jump downward so that $y$ jumps downward to an optimal target value. Optimal investment behavior is completely characterized in terms of $y$ and the trigger and target values. In this paper we provide 
analytic approximations to the trigger and target values which allow us to study the impact of fundamental parameters on investment.

In the next section, we set up the firm's environment and solve the instantaneous optimization problem characterizing employment and factor utilization for a given value of the capital stock. We characterize optimal investment in Section III and present the analytic approximations for the investment trigger and target in Section IV. These results are used in Section V to analyze the expected duration of the Marshallian short run. In Section VI, we then show how these findings imply labor hoarding and short-run increasing returns to labor and examine the dynamics of employment and factor utilization when the capital stock is adjusted. Finally, we consider how the state variable governing investment might be observed in practice.

\section{The Instantaneous Determination of Utilization and Employment}

The firm maximizes the present value of expected profits over an infinite horizon. It derives its revenue from selling output $Q$ at price $P$ in a market in which it faces a downward-sloping demand curve. We specify an isoelastic demand curve

$$
Q^{d}=\left(\frac{P}{X_{1}}\right)^{-\varepsilon},
$$

with finite price elasticity of demand $\varepsilon>1$. The demand curve in equation (1) is subject to the shock $X_{1}$ which evolves according to a geometric Brownian motion. (Although the various shocks and decision variables change over time, we suppress the time subscripts on these variables where we can do so without loss of clarity.) This shock causes the 
demand curve to shift upward or downward, and the price associated with a given quantity of output is proportional to $X_{1}$.

Output, $Q$, is produced using two factors of production, capital, $K$, and labor, $L$. The firm chooses how much capital and labor to employ, as well as the level of factor utilization, $u$. Output is produced according to a Cobb-Douglas production function

$$
Q=X_{2} u^{v} K^{\beta} L^{\alpha}, v \leq \alpha+\beta \leq 1,
$$

where $X_{2}$ is a productivity shock that evolves according to geometric Brownian motion. The specification in equation (2) allows for either constant returns to scale $(\alpha+\beta=1)$ or decreasing returns to scale $(\alpha+\beta<1)$ in capital and labor. In the special case in which $v$ $=\alpha+\beta$, output is a function of capital services $u K$ and labor services $u L$.

The demand and production functions imply the revenue function

$$
R(X, u, K, L)=X\left(u^{v} K^{\beta} L^{\alpha}\right)^{1-\frac{1}{\varepsilon}},
$$

where $X \equiv X_{1} X_{2}^{1-\frac{1}{\varepsilon}}$ is the shock to the revenue function. Because $X_{1}$ and $X_{2}$ each follows a geometric Brownian motion, the revenue shock, $X$, also follows a geometric Brownian motion. We specify this geometric Brownian motion as

$$
d X=\mu_{X} X d t+\sigma_{X} X d z_{X},
$$

where $d z x$ is the increment of a standard Wiener process.

The operating profit of the firm equals revenue less the operating costs incurred by utilizing labor and capital: 


$$
\pi(X, u, K, L)=R(X, u, K, L)-w u^{\eta} L-m u^{\rho} K, \quad \frac{v}{\alpha}>\eta \geq 1, \rho \geq 1 .
$$

Operating costs are increasing and convex in the factor utilization rate, $u$, which is the common utilization rate of capital and labor. ${ }^{4,5}$ The firm pays a unit cost, $m u^{\rho}$, to utilize capital where $m$ is the unit cost of operating capital when $u=1$. We will refer to $m$ as the standardized unit operating cost of capital. The unit operating cost $m u^{\rho}$ may represent materials, maintenance, or utilities expenses, for example, and is an increasing and (weakly) convex function of utilization. Similarly, total labor $\operatorname{costs}, w u^{\eta} L$, depend on utilization, so that the marginal labor cost is an increasing and (weakly) convex function of factor utilization. This specification may represent the payment of an overtime premium, for example, or the cost of materials or other inputs used by employed labor. The factor price $w$ is the standardized unit cost of labor, which is the cost per unit of labor evaluated at $u=1$. We assume that the standardized operating costs of labor and capital follow geometric Brownian motion processes ${ }^{6}$ according to

$$
d w=\mu_{w} w d t+\sigma_{w} w d z_{w}
$$

and

$$
d m=\mu_{m} m d t+\sigma_{m} m d z_{m},
$$

where $d z_{w}$ and $d z_{m}$ are increments to two Wiener processes.

In addition to operating costs, the firm pays acquisition and fixed costs when the capital stock is increased; the exact form of these costs is specified below when we consider the dynamics of the capital stock. We assume that investment is irreversible, so that the capital stock falls only through depreciation. 
We first consider the firm's optimal choices of utilization and labor for a given capital stock, and then turn, in Section III, to the optimal investment decision. Static firstorder conditions govern the firm's choice of utilization and employment since there are no costs of adjustment associated with utilization or labor. The first-order conditions for utilization and labor, respectively, are given by

$$
v\left(1-\frac{1}{\varepsilon}\right) R=\eta w u^{\eta} L+\rho m u^{\rho} K
$$

and

$$
\alpha\left(1-\frac{1}{\varepsilon}\right) R=w u^{\eta} L
$$

Use equations (7) and (8) to substitute for the operating costs of labor and capital in equation (5) to obtain

$$
\pi=\frac{\Delta}{\rho} R, \quad \text { where } \Delta \equiv \rho-[v+\alpha(\rho-\eta)]\left(1-\frac{1}{\varepsilon}\right)>0 .^{7}
$$

We derive a relationship between the labor-capital ratio and the level of utilization by taking the ratio of equation (7) to equation (8) to obtain

$$
\frac{L}{K}=\frac{\rho \alpha}{v-\alpha \eta} u^{\rho-\eta} \frac{m}{w}
$$

Notice that when $\rho=\eta$, the labor-capital ratio is proportional to $\frac{m}{w}$ and invariant to the utilization rate, $u$. In this case, the ratio of standardized operating costs, $\frac{w L}{m K}$, is constant. 
The effects of the two factor prices, $w$ and $m$, on employment and utilization can be evaluated by defining the operating cost index $C$ and the relative price $\omega$ as

$$
C \equiv w^{\frac{\alpha}{\alpha+\beta}} m^{\frac{\beta}{\alpha+\beta}}
$$

and

$$
\omega \equiv \frac{m}{w}
$$

Substituting these measures into the first-order conditions for labor and utilization, we derive expressions for optimal utilization and employment

$$
\begin{aligned}
& u=A_{u}\left(\frac{C}{X}\right)^{-\frac{1}{\Delta}} \omega^{-\frac{\alpha}{\alpha+\beta} \frac{1-\theta}{\rho}} K^{-\frac{1-\theta}{\rho}}, \text { and } \\
& \frac{L}{K}=A_{\ell}\left(\frac{C}{X}\right)^{-\frac{1}{\Delta}(\rho-\eta)} \omega^{1-(\rho-\eta) \frac{\alpha}{\alpha+\beta} \frac{1-\theta}{\rho}} K^{-\frac{(1-\theta)(\rho-\eta)}{\rho}},
\end{aligned}
$$

where $^{8}$

$$
\begin{gathered}
\theta \equiv \frac{1}{\Delta}\left(1-\frac{1}{\varepsilon}\right)[\beta \rho-(v-\alpha \eta)], \quad 0<\theta<1, \\
A_{u} \equiv\left(1-\frac{1}{\varepsilon}\right)^{\frac{1}{\Delta}}(v-\eta \alpha)^{\frac{\rho-\alpha(\rho-\eta)\left(1-\frac{1}{\varepsilon}\right)}{\rho \Delta}} \alpha^{\frac{\alpha\left(1-\frac{1}{\varepsilon}\right)}{\Delta}} \rho^{\frac{\alpha\left(1-\frac{1}{\varepsilon}\right)-1}{\Delta}}>0,
\end{gathered}
$$

and

$$
A_{\ell} \equiv \frac{\alpha \rho}{v-\alpha \eta} A_{u}^{\rho-\eta}>0
$$

Optimal utilization is decreasing in the capital stock and decreasing in the two cost measures, $\frac{C}{X}$ and $\omega$. The first measure, $\frac{C}{X}$, is most easily interpreted in the absence of productivity shocks ( $X_{2} \equiv$ a constant). In this case, $X$ is proportional to the demand 
shock $X_{1}$ in equation (1) which is proportional to the price of output. Thus, $\frac{C}{X}$ can be interpreted as an operating cost index measured in real terms. In the case with productivity shocks but no demand shocks $\left(X_{1} \equiv\right.$ a constant $), X$ is proportional to productivity $X_{2}$. An increase in productivity reduces the effective cost of factors and reduces the ratio $\frac{C}{X}$. In the most general case with both demand and productivity shocks, we interpret the ratio $\frac{C}{X}$ as an index of real effective operating costs. As shown in equation (12), optimal utilization is a decreasing function of the real effective operating cost index.

Utilization is also decreasing in the relative operating cost of capital compared to labor, $\omega \equiv m / w$, owing to the quasi-fixity of capital. During times in which the capital stock is not adjusted, the firm decreases utilization if capital becomes relatively more expensive to operate. This result is independent of the value of $v$; because the firm cannot shed capital, when capital becomes more expensive to operate (relative to labor), it is used less intensively.

A decrease in the index of real effective operating cost, $C / X$, will cause the optimal labor-capital ratio to rise, remain unchanged, or fall depending on whether $\rho-\eta$ is positive, zero, or negative. This dependence on the sign of $\rho-\eta$ is apparent from equation (10) which indicates that for a given ratio of standardized operating costs, $\omega \equiv$ $m / w$, the optimal labor-capital ratio is proportional to $u^{\rho-\eta}$. Recall that a drop in the index of real effective operating costs $C / X$ increases optimal utilization $u$. If $\rho=\eta$, there is no change in the optimal labor-capital ratio. However, if $\rho>\eta$, the increase in utilization 
causes the unit operating cost of capital to rise by more than the unit operating cost of labor rises, so the firm shifts away from capital toward labor, and the optimal laborcapital ratio increases. In contrast, if $\rho<\eta$, the increase in utilization causes the unit operating cost of labor to increase by more than the unit operating cost of capital increases; thus, the firm shifts away from labor toward capital, and the optimal laborcapital ratio falls.

Although the sign of the response of the optimal labor-capital ratio to a change in $C / X$ depends on the sign of $\rho-\eta$, an increase in the relative operating cost of capital $\omega \equiv$ $\mathrm{m} / \mathrm{w}$ unambiguously increases the optimal labor-capital ratio as the firm shifts toward labor.9

We can gain some insight into the utilization-employment margin by considering the optimal response to an increase in the demand variable $X_{1}$ (holding productivity $X_{2}$ fixed) in the case in which $\rho=\eta>1$. When $X_{1}$ increases, the firm increases its output. With the capital stock fixed, output can be increased by increasing either utilization $u$ or employment $L$. A one percent increase in $u$ is more costly than a one percent increase in $L$, but it also increases output by more than does a one percent increase in $L$. Specifically, the elasticity of the operating cost of labor with respect to $u$ is $\eta>1$, and the elasticity of the operating cost of labor with respect to $L$ is one. The elasticity of output with respect to $u$ is $v$ and the elasticity of output with respect to $L$ is $\alpha$. Taking account of the effects on both costs and output, the restriction that $v \eta>\alpha$ implies that it is optimal to respond to the increase in $X \equiv X_{1} X_{2}^{1-\frac{1}{\varepsilon}}$ by increasing utilization. In fact, for the case in which $\rho=$ 
$\eta>1$, equations (12) and (13) imply that the optimal response to an increase in $X$ is for employment $L$ to remain unchanged and for utilization to increase.

Substituting the expressions for optimal utilization and employment from equations (12) and (13) into the revenue function in equation (3), we obtain

$$
\begin{gathered}
R[X, u(C, X, \omega, K), K, L(C, X, \omega, K)]=A_{R} X\left(\frac{C}{X}\right)^{1-\frac{\rho}{\Delta}} \omega^{\frac{\alpha}{\alpha+\beta} \frac{1-\frac{1}{\varepsilon}}{\Delta}[\alpha \eta \eta+\beta \rho-v]} K^{\theta}, \text { where } \\
A_{R} \equiv\left(1-\frac{1}{\varepsilon}\right)^{\frac{1}{\Delta}\left(1-\frac{1}{\varepsilon}\right)(\gamma+\alpha\{\rho-\eta)]}\left(\frac{v-\alpha \eta}{\rho}\right)^{\frac{1}{\Delta}\left(1-\frac{1}{\varepsilon}\right)(v-\alpha \eta]} \alpha^{\frac{\rho \alpha}{\Delta}\left(1-\frac{1}{\varepsilon}\right)}>0 .
\end{gathered}
$$

The terms involving $X, C$, and $\omega$ in this revenue function can be combined into a single variable defined by

$$
Z \equiv\left[w^{-\alpha \rho\left(1-\frac{1}{\varepsilon}\right)} m^{-(v-\alpha \eta)\left(1-\frac{1}{\epsilon}\right)} X^{\rho}\right]^{\frac{1}{(1-\theta) \Delta}} .
$$

The variable $Z$ is the product of geometric Brownian motions and hence is also a geometric Brownian motion ${ }^{10}$ which we characterize as

$$
d Z=\mu_{\mathrm{z}} Z d t+\sigma_{\mathrm{z}} Z d z_{\mathrm{Z}},
$$

where the instantaneous drift $\mu_{Z}$ and the instantaneous standard deviation $\sigma_{Z}$ are functions of the parameters of the geometric Brownian motions for $X, m$, and $w$. We assume that these parameters satisfy $\mu_{z}>\frac{1}{2} \sigma_{z}^{2}-\delta$.

The variable $Z$ allows us to write the revenue function simply as 


$$
\hat{R}(Z, K)=A_{R} Z^{1-\theta} K^{\theta}
$$

Substituting equation (17) into equation (9) yields an expression for maximized operating profits

$$
\pi(Z, K)=A_{n} Z^{1-\theta} K^{\theta}, \text { where } A_{n} \equiv \frac{\Delta}{\rho} A_{R}>0
$$

With this characterization of operating profits, which incorporates optimal utilization and employment, we now turn to adjustment of the capital stock.

\section{Optimal Investment}

Unlike labor and utilization which can be costlessly and instantaneously adjusted, capital is costly to adjust. Specifically, we assume that in addition to the purchase price of capital, the firm incurs a fixed cost each time that it purchases capital. Moreover, we assume that capital investment is irreversible and that capital depreciates at a constant rate $\delta>0$. Therefore, the firm must look to the future when deciding when and how much capital to purchase. In this section we study the firm's intertemporal investment problem.

We assume that the firm is risk-neutral and maximizes the present value of expected cash flows discounted at a constant positive discount rate $r>\mu_{\mathrm{z} \cdot}{ }^{11}$ The firm can buy capital at a constant price $p>0$. In addition, whenever the firm purchases capital it pays a fixed cost $Z_{t} F>0$. Rather than assume that the fixed cost is simply constant over time, we specify the fixed cost to be proportional to $Z_{t}$ which is a linearly homogeneous index of the various prices in the model. This specification prevents the fixed cost from 
becoming trivial if the various prices in the model become very large, and prevents the fixed cost from being overwhelming if the various prices in the model become small. This specification also simplifies the analysis by making the value of the firm a linearly homogeneous function of $Z_{t}$ and $K_{t}{ }^{12}$

The value of the firm is

$$
V\left(K_{t}, Z_{t}\right) \equiv \max _{\left\{t_{i}, \Delta K_{t_{i}} 20\right\}} E_{t}\left\{\int_{0}^{\infty} A_{\pi} Z_{t+s}^{1-\theta} K_{t+s}^{\theta} e^{-r s} d s-\sum_{i=1}^{\infty} e^{-r\left(t_{i}-t\right)}\left(p \Delta K_{t_{i}}+Z_{t_{i}} F\right)\right\}
$$

The integral on the right hand side of equation (19) is the present value of operating profits from time $t$ onward. The summation on the right hand side of this equation is the present value of investment costs in the future. Because of the fixed cost of investment, it is optimal to invest at distinct points of time $t_{i}, i=1,2,3, \ldots$. When the firm invests at time $t_{i}$, it buys a finite amount of capital $\Delta K_{t_{i}}$. The cost of purchasing this capital is $p \Delta K_{t_{i}}$ and the firm also incurs a fixed cost $Z_{t_{i}} F$. The firm's investment problem is to choose the times to buy capital, $t_{i}$, and the amounts of capital, $\Delta K_{t_{i}}$, to buy.

The value function in equation (19) is homogeneous of degree one in $Z$ and $K$ so that the value function can be written as

$$
V(K, Z)=K v(y) \text { where } y \equiv Z / K
$$

The ratio $y$ is a scalar variable that is a sufficient statistic for the firm's investment decision. When gross investment is zero, $y$ evolves as a geometric Brownian motion with instantaneous drift $\mu_{y} \equiv \mu_{z}+\delta$ and standard deviation $\sigma_{y} \equiv \sigma_{z}$.

Because the value function is homogeneous of degree one in $Z$ and $K$, the marginal value of capital $V_{K}(K, Z)$ is homogeneous of degree zero in $Z$ and $K$. Let $q(y) \equiv$ 
$V_{K}(K, Z)$ denote the marginal value of capital. Optimality implies that the marginal unit of capital earns a return equal to the required return $r q(y)$. The return earned by the marginal unit of capital has three components: (1) the marginal profit of capital, $\theta A_{\pi} y^{1-\theta}$; (2) the expected capital gain on the marginal valuation of a unit of capital, $(1 / d t) E\{d q\}$; and (3) a loss equal to $\delta q$ arising from the physical depreciation of capital. Using Ito's Lemma to evaluate the capital gain on the marginal valuation of capital, and setting the required return equal to the sum of the three components of the return on capital yields

$$
(r+\delta) q(y)=h y^{\gamma}+\mu_{y} y q^{\prime}(y)+\frac{1}{2} \sigma_{y}^{2} y^{2} q^{\prime \prime}(y) .
$$

where $h \equiv \theta A_{\pi}$. and $\gamma \equiv 1-\theta$.

The solution to the differential equation (21) involves the roots of the following quadratic equation (see, for example, Dixit and Pindyck (1994))

$$
v(\xi) \equiv-\frac{1}{2} \sigma_{y}^{2} \xi^{2}-\left(\mu_{y}-\frac{1}{2} \sigma_{y}^{2}\right) \xi+r+\delta=0 .
$$

Note that $u(\xi)$ is strictly concave, $v(0)=r+\delta>0$, and $\nu(1)=r+\delta-\mu_{y}>0$. Thus, $v(\gamma)>0$, and $\mathcal{Q}(\xi)=0$ has two distinct roots, $\alpha_{P}>0$ and $\alpha_{N}<0$, which satisfy

$$
\alpha_{N}<0<\gamma<1<\alpha_{p} .
$$

The roots also satisfy the following equation

$$
v(\gamma)=\frac{\left(\gamma-\alpha_{P}\right)\left(\gamma-\alpha_{N}\right)}{\alpha_{P} \alpha_{N}}(r+\delta) .
$$

The general solution to the differential equation in (21) is 


$$
q(y)=H y^{\gamma}+D y^{\alpha_{N}}+E y^{\alpha_{p}} \text { where } H \equiv \frac{h}{v(\gamma)}>0,
$$

and $D$ and $E$ are constants to be determined. We will show below that optimality implies that the ratio $y$ is a controlled geometric Brownian motion. Specifically, the firm never allows the ratio $y$ to exceed a given trigger value. However, $y$ may take on arbitrarily small values. As $y$ becomes arbitrarily small, the value of the firm and the marginal value of capital become small, yet $\lim _{y \rightarrow 0} y^{\alpha_{N}}=\infty$. In order for $\lim _{y \rightarrow 0} q(y)$ to be finite, $D$ must be zero. Thus, the general solution to the differential equation is

$$
q(y)=H y^{\gamma}+E y^{\alpha_{P}}
$$

where the constant $E$ still remains to be determined.

\section{III.1 The Boundary Conditions}

The firm must choose when and how much capital to purchase. Most of the time, the firm does not invest. During these time intervals of zero investment, the ratio $y$ follows a geometric Brownian motion. When $y$ increases, the marginal profit of capital increases and investment looks more attractive. If $y$ gets high enough, then it is optimal for the firm to incur the fixed cost and to buy capital. Let $b$ be the trigger value of $y$ that induces the firm to buy capital. When the firm buys capital, it buys a discrete amount of capital, thereby causing the denominator of $y \equiv Z / K$ to jump upward, and hence causing the ratio $y$ to jump downward to a target value that we denote as $c<b$.

The values of the trigger $b$, the target $c$, and the constant $E$ are determined by the boundary conditions: the value-matching and smooth-pasting conditions. According to the value-matching condition, the increase in the value of the firm when it acquires new 
capital is equal to the fixed cost that the firm incurs when it undertakes the investment.

We write this condition as

$$
\int_{Z / b}^{Z / c}\left[V_{K}(K, Z)-p\right] d K=Z F
$$

The smooth-pasting conditions require that the marginal valuation of capital $q(y)$ is equal to the purchase price of capital $p$ immediately before and immediately after investing. We write these conditions as

$$
\begin{aligned}
& q(c)=p \\
& q(b)=p
\end{aligned}
$$

The smooth-pasting conditions $(28 \mathrm{a}, \mathrm{b})$ imply that $q(b)=q(c)$ which along with equation (26) implies

$$
H\left(b^{\gamma}-c^{\gamma}\right)+E\left(b^{\alpha_{P}}-c^{\alpha_{P}}\right)=0 .
$$

Recalling that $H>0$ and that $b \neq c$, equation (29) immediately implies that $E<0$. We can obtain an expression for $E$ by using the general solution in equation (26) along with the smooth-pasting conditions $(28 \mathrm{a}, \mathrm{b})$ to obtain

$$
\frac{p-H b^{\gamma}}{b^{\alpha_{p}}}=E=\frac{p-H c^{\gamma}}{c^{\alpha_{p}}}
$$

Next rewrite equation (30) to obtain an equation relating the trigger $b$ and the target $c$

$$
J^{1}(b, c) \equiv\left(p-H c^{\gamma}\right) b^{\alpha_{p}}-\left(p-H b^{\gamma}\right) c^{\alpha_{p}}=0
$$


We have used the smooth-pasting conditions to derive equation (30) relating $b$ and $c$. Next we use the value-matching condition to obtain a second equation relating $b$ and $c$. Together these two equations will allow us to solve for the trigger and target points.

We perform the integration in the value-matching condition in equation (27) by using a change-of-variable transformation. Specifically, we replace $V_{K}(K, Z)$ by the expression for $q(y)$ in equation (26), use the fact that for a given value of $Z, d K=$ $\left(Z / y^{2}\right) d y$, and perform the integration in equation (27) to obtain

$$
\frac{1}{\gamma-1} H y^{\gamma-1}+\frac{1}{\alpha_{p}-1} E y^{\alpha_{p}-1}+\left.p y^{-1}\right|_{c} ^{b}=F
$$

Next use equation (30) to eliminate $E$ from equation (32) and rearrange the equation to obtain

$$
J^{2}(b, c ; F) \equiv \frac{\alpha_{P}-\gamma}{(\gamma-1)\left(\alpha_{P}-1\right)} H\left(b^{\gamma-1}-c^{\gamma-1}\right)+\left(\frac{\alpha_{P}}{\alpha_{P}-1}\right) p\left(b^{-1}-c^{-1}\right)-F=0
$$

We have reduced the firm's investment problem to the determination of two constants: the trigger value $b$ and the target value $c$. We have developed a two-equation system characterizing these two values. The smooth-pasting conditions led to the equation $J^{1}(b, c)=0$, and the value-matching condition led to the equation $J^{2}(b, c ; F)=0$. In the absence of a fixed cost, i.e., when $F=0$, these equations are satisfied by $b=c=\xi$ for any $\xi>0$. However, in the interesting case with $F>0$, we know of no analytic solution of this bivariate system. Depending on the issue being studied, we could either solve this system numerically ${ }^{13}$ or use an analytic approximation to the solution. We pursue the second strategy in the next section. 


\section{Approximate Solutions for the Investment Trigger and Target}

In order to derive approximate solutions for the bivariate system $J^{1}(b, c)=0$ and $J^{2}(b, c ; F)=0$, we calculate Taylor series expansions of the functions $J^{1}(b, c)$ and $J^{2}(b, c ; F)$, set these expressions equal to zero, and solve for the values of $b$ and $c$. To implement this strategy, we need to choose a point around which to expand, and we need to choose the order of the expansion. As we discuss below, we expand around the values of $b$ and $c$ that would hold when the fixed cost is arbitrarily small. As for the order of the Taylor expansion, simplicity dictates using the smallest order feasible. However, neither a first-order nor a second-order Taylor series approximation is sufficient to implement this procedure. Thus, we use a third-order expansion.

In the absence of a fixed cost of investment, the firm's investment problem is a standard problem of irreversible investment under uncertainty. The solution to this problem is to buy capital whenever it is needed to prevent the marginal profit of capital $h y^{\gamma}$ from increasing above an appropriately defined user cost of capital. Equivalently, the solution is to buy capital whenever necessary to prevent the ratio $y$ from rising above a trigger value $\phi$. (Formally, in the absence of fixed costs, the trigger point $b$ and the target point $c$ are both equal to $\phi$, which is defined below.)

In the absence of a fixed cost of investment, the trigger value $\phi$ of the state variable $y$ satisfies the following condition ${ }^{14}$

$$
h \phi^{\gamma}=\left(1-\frac{\gamma}{\alpha_{N}}\right)(r+\delta) p
$$


The left-hand side of equation (34) is the marginal profit of capital when $y$ equals the trigger value $\phi$. The right-hand side of this equation is the user cost of capital for irreversible investment in the absence of a fixed cost. Since $\gamma$ is positive and the root $\alpha_{N}$ is negative, the user cost of capital in equation (34) exceeds the user cost of capital with costless reversibility, $(r+\delta) p$, derived by Jorgenson (1963).

Now expand the functions $J^{1}(b, c)$ and $J^{2}(b, c ; F)$ around $b=c=\phi$ and $F=0$.

Define $\tilde{b} \equiv b-\phi$ and $\tilde{c} \equiv c-\phi$. Because all first partial derivatives of these functions are zero at the point of expansion, we compute second-order Taylor series approximations to these functions

$$
\begin{aligned}
& J^{1}(b, c) \cong \frac{1}{2}\left(\gamma-\alpha_{P}\right) \gamma H \phi^{\gamma+\alpha_{P}-2}\left(\tilde{b}^{2}-\tilde{c}^{2}\right) \\
& J^{2}(b, c ; F) \cong \frac{1}{2} \frac{\alpha_{P}-\gamma}{\alpha_{P}-1} \gamma H \phi^{\gamma-3}\left(\tilde{b}^{2}-\widetilde{c}^{2}\right)-F
\end{aligned}
$$

The strategy for calculating $\tilde{b}$ and $\tilde{c}$ is to set the right-hand sides of equations (35a,b) equal to zero and solve for $\tilde{b}$ and $\tilde{c}$ given $F$. Setting the right-hand side of equation (35a) equal to zero yields $\left(\tilde{b}^{2}-\tilde{c}^{2}\right)=0$. Substituting this result into the right hand side of equation (35b) and setting the right hand side equal to zero yields $-F=0$, which is a contradiction whenever there are positive fixed costs. Thus, we must go to a third-order approximation. The third-order approximation and the calculation of the solution values for $\tilde{b}$ and $\tilde{c}$ are contained in Appendix $\mathrm{C}$ where it is shown that for small values of $F$ 


$$
\tilde{b} \cong\left(\frac{1.5}{\alpha_{P} \gamma} \frac{F}{p} \phi\right)^{1 / 3} \phi>0
$$

and

$$
\tilde{c} \equiv-\frac{3 \phi}{3 \phi-\left(\gamma-3-2 \alpha_{P}\right) \tilde{b}} \tilde{b}<0
$$

It follows from equation (36b) that $\tilde{c} \cong-\tilde{b}$ for small values of $\tilde{b}$. Also equations $(36 \mathrm{a}, \mathrm{b})$ imply that $\tilde{b}+\tilde{c}>0$ and hence that $|\tilde{b}|>|\tilde{c}|$.

We interpret $\tilde{b} \cong\left(\frac{15}{\alpha_{P} \gamma} \frac{F}{p} \phi\right)^{1 / 3} \phi$ in equation (36a) as a wedge between the trigger value of $y$ in the absence of fixed costs and the trigger value in the presence of small fixed costs. The relationship between this wedge and the fixed cost is cubic. ${ }^{15}$ It is straightforward to calculate the derivative of this wedge with respect to the fixed cost $F$. This derivative is infinite when evaluated at $F=0$ which means that even a tiny fixed cost will substantially increase the trigger value of $y$ above $\phi$.

The expression for the wedge in equation (36a) contains the term $F \phi / p$. Recall that $\phi$ is the ratio of $Z$ to $K$ when investment takes place under irreversibility without a fixed cost. Let $Z^{*}$ and $K^{*}$ be the values of these variables at an instant of investment. Then $\phi=Z^{*} / K^{*}$ and $F \phi / p=F Z^{*} /\left(p K^{*}\right)$ is the ratio of the fixed cost to the replacement cost of the capital stock if investment were to take place according to the optimal rule for irreversible investment with zero fixed cost. The wedge in equation (36a) is an increasing function of the ratio of the fixed cost to the replacement cost of capital. 


\section{The Duration of Time Between Successive Instants of Investment}

We have shown that investment takes place at discrete instants of time. Now we analyze the duration of time between successive instants at which investment occurs.

Recall that the state variable $y$ equals $c$ immediately after investment takes place, and that the next instant of investment occurs when $y$ first reaches the trigger value $b$. Thus, the duration of time between successive instants of investment is the first passage time of $y$ from the target point $c$ to the trigger point $b$. The expected value of the first passage time is a simple application of the following Lemma (the proofs of the Lemmas and Propositions in this section are in Appendix D):

Lemma 1: Let $d y / y=\mu_{y} d t+\sigma_{y} d z$, where $\mu_{y}>\sigma_{y}^{2} / 2$ and $d z$ is the increment to a standard Wiener process. The expected value of the first passage time from $y_{0}$ to $y_{1}>y_{0}$ is $\frac{\ln \left(y_{1} / y_{0}\right)}{\mu_{y}-(1 / 2) \sigma_{y}^{2}}$

Let $T(c, b)$ be the first passage time of $y$ from the target point $c$ to the trigger point $b$. The following corollary is an immediate implication of Lemma 1.

Corollary 1 to Lemma 1: $\quad E\{T(c, b)\}=\frac{\ln (b / c)}{\mu_{y}-(1 / 2) \sigma_{y}{ }^{2}}$

Lemma 2: For small fixed cost $F, \ln \left(\frac{b}{c}\right) \cong 2\left(\frac{1.5}{\alpha_{p} \gamma} \frac{F}{p} \phi\right)^{1 / 3}$. 
Lemma 2 indicates that the (geometric) distance between the target point $c$ and the trigger $b$ is an increasing function of $F$.

We use Lemmas 1 and 2 to calculate the expected duration of time between successive instants of investment.

Proposition 1: For small fixed cost $F, \quad E\{T(c, b)\} \cong \frac{2}{\mu_{y}-(1 / 2) \sigma_{y}^{2}}\left(\frac{1.5}{\alpha_{p} \gamma} \frac{F}{p} \phi\right)^{1 / 3}$.

It follows immediately from Proposition 1 that an increase in the fixed cost $F$ increases the expected duration of time between successive instants of investment. The reason is that an increase in the fixed cost induces the firm to widen the distance between the target point $c$ and the trigger point $b$ (Lemma 2 ) in order to delay the occurrence of the next time that the firm must pay the fixed cost.

Next we consider the effect of an increase in the instantaneous variance $\sigma_{y}^{2}$ on the expected duration of time between successive instants of investment. ${ }^{16}$ This effect depends on two factors. First, an increase in $\sigma_{y}^{2}$ increases the expected value of the first passage time between two given points, as described below in Corollary 2 to Lemma 1 . Second, for a small fixed cost $F$, an increase in $\sigma_{y}{ }^{2}$ widens the gap between the target point $c$ and the trigger point $b$. This effect, which is captured in Proposition 2 below, reinforces the first effect.

Inspection of Lemma 1 immediately proves the following corollary 
Corollary 2 to Lemma 1: An increase in $\sigma_{y}^{2}$ increases $\frac{1}{\mu_{y}-(1 / 2) \sigma_{y}{ }^{2}}$ and hence increases the expected first passage time of $y$ from $y_{0}$ to $y_{1}>y_{0}$.

This corollary applies to the expected first passage time from a given value $y_{0}$ to a given value $y_{1}$. However, an increase in the instantaneous variance $\sigma_{y}^{2}$ changes the trigger and target points.

Proposition 2: For small fixed cost $F$, an increase in $\sigma_{y}^{2}$ increases $\ln (b / c)$, the geometric distance between the target point $c$ and trigger point $b$.

Proposition 3: For small fixed cost $F$, an increase in $\sigma_{y}^{2}$ increases $E\{T(c, b)\}$, the expected duration of time between successive instants of investment.

An increase in $\sigma_{y}^{2}$ increases $E\{T(c, b)\}$ for two reasons. First, an increase in $\sigma_{y}^{2}$ increases the expected first passage time from a given value to another given value (Corollary 2 to Lemma 1). Second, an increase in $\sigma_{y}^{2}$ widens the distance between the target point $c$ and the trigger point $b$ (Proposition 2).

We have derived two results that may seem to be contradictory. Corollary 2 to Lemma 1 indicates that for given values of $c$ and $b$, an increase in $\sigma_{y}{ }^{2}$ increases the expected first passage time from $c$ to $b$. Thus, for given $c$ and $b$, the expected time until the next payment of the fixed cost rises with $\sigma_{y}^{2}$, yet Proposition 2 states that the firm responds by widening the distance between $c$ and $b$ (further increasing the first passage 
time). However, one might expect the firm instead to narrow the gap between $c$ and $b$ when the expected first passage time rises. This apparent contradiction is resolved by the following proposition.

Proposition 4: For given $y_{1}>y_{0}$, if $r+\delta>\mu_{y}>(1 / 2) \sigma_{y}^{2}$, an increase in $\sigma_{y}^{2}$ increases $E\left\{e^{-(r+\delta) T\left(y_{0}, v_{1}\right)}\right\}$, the expected present value (discounted at rate $r+\delta$ ) of a unit paid at the first passage time of $y$ from $y_{0}$ to $y_{1}$.

Proposition 4 shows that if the target point $c$ and the trigger point $b$ were unchanged, an increase in $\sigma_{y}^{2}$ would increase the expected present value of a unit to be paid when the stochastic process first reaches $b$ - despite the increase in the expected length of time it takes to reach $b$. The firm responds to this increase in expected present value of costs by widening the band between the target point $c$ and the trigger point $b$, thereby delaying the next payment of investment costs.

\section{Employment and Utilization with Quasi-Fixed Capital}

In the previous section we showed that the dynamic behavior of the capital stock is governed by the state variable $y \equiv Z / K$ which is a controlled geometric Brownian motion. When $y$ is below the trigger value $b$, the firm does not invest and the capital stock depreciates at the rate $\delta$. Values of $y$ below the trigger are often described as comprising a "range of inaction." While it is true that gross investment is zero in the range of inaction, utilization and (except when $\rho=\eta$ ) employment are continuously adjusted in response to changes in demand, productivity, and factor prices. We analyzed 
this behavior in section II where the capital stock was held fixed. Here, we further draw out the implications for employment in the "range of inaction". We also examine the joint behavior of capital, labor, and utilization when the state variable $y$ reaches its trigger value $b$, so that the firm buys capital and adjusts utilization and employment. Finally, we discuss how to measure investment state variable, $y$, which determines the timing of capital expenditures.

\section{VI.1 Short-run Increasing Returns to Labor and Labor Hoarding}

Optimal investment behavior implies that there are discrete intervals of time during which the firm does not purchase capital. The interval of time until the next purchase of capital is a natural definition of the short run since it is the period of time during which the capital stock is fixed, except for exogenous depreciation. During the short run, optimal behavior is characterized by the utilization and the employment decisions in equations (12) and (13). Here we consider the response of utilization and employment to changes in demand $X_{1}$, productivity $X_{2}$, and the operating cost index $C$ in the short run. For clarity, we confine attention to cases in which the relative price ratio $\omega$ $\equiv m / w$ is fixed in the short run.

The effects of $X_{1}, X_{2}$, and $C$ on optimal factor utilization and optimal employment are completely captured by the real effective operating cost index $C / X$. Let $\varepsilon_{u, C / X}$ be the short-run elasticity of optimal utilization with respect to $C / X$, and let $\varepsilon_{L, C X}$ be the shortrun elasticity of optimal employment with respect to $C / X$. Inspection of equations (12) and (13) and reveals that

$$
\varepsilon_{u, C / X}=-\frac{1}{\Delta}, \text { and }
$$




$$
\varepsilon_{L, C / X}=-\frac{\rho-\eta}{\Delta}
$$

Now consider the effect of a $1 \%$ decrease in the real effective operating cost index $C / X$, while holding productivity $X_{2}$ fixed. ${ }^{17}$ Using the production function in equation (2) and the elasticities in equations (37) and (38), it is straightforward to show that output increases by $\frac{1}{\Delta}[v+(\rho-\eta) \alpha]$ percent while labor increases by only $\frac{\rho-\eta}{\Delta}$ percent. If an observer were to ignore the role of factor utilization and attribute the entire increase in output to the increase in labor, the estimate of the short-run elasticity of output with respect to labor would be

$$
\varepsilon_{Q, L}=\alpha+\frac{v}{\rho-\eta}
$$

If $\rho \geq \eta$, which is the case in which labor increases while output increases, the estimated short-run elasticity of output with respect labor in equation (39) exceeds the "true" elasticity $\alpha$, perhaps by a substantial amount. The "bias" in the estimated elasticity, $v(\rho-$ $\eta$ ), can be large enough to make the estimated short-run elasticity of output with respect to labor greater than one. Thus, the bias introduced by ignoring the role of factor utilization can potentially account for the finding of short-run increasing returns to labor reported in the literature. Burnside, Eichenbaum, and Rebelo (1993), among others, consider this possibility by allowing for variable labor effort.

Now consider the extreme case in which $\rho=\eta$. To focus on labor hoarding, we consider a drop in demand that increases the real effective operating cost index $C / X$ by one percent. With $\rho=\eta$, equation (38) indicates that the firm does not change the 
amount of labor it employs. Nevertheless, the amount of output falls by $v \Delta$ percent (so the estimated elasticity in equation (39) is infinite) by reducing factor utilization by $1 / \Delta$ percent. This phenomenon, in which the firm does not reduce its employment of labor in the face of a drop in demand and consequent drop in output, is known as labor hoarding.

Labor hoarding is often attributed to costs associated with firing workers and then hiring and training workers when demand picks up in the future (Bentolila and Bertola (1990), Hopenhayn and Rogerson (1993)), or to implicit labor contracts and other labor market institutions (Fay and Medoff (1985)). Optimal behavior in our model will display labor hoarding in response to short-run changes in demand even though there are no direct costs associated with firing, hiring, or training labor. The labor hoarding exhibited here results from the quasi-fixity of capital arising from the fixed cost of investment (and from the irreversibility of investment). In effect, these costs of adjusting the capital stock induce "capital hoarding" and the fact that the optimal labor-capital ratio remains unchanged induces labor hoarding as a counterpart to capital hoarding. The fact that labor hoarding can arise without direct costs of adjusting the level of employment casts doubt on any attempts to measure the costs of adjusting employment simply by focusing on the behavior of employment without looking at other factors of production. ${ }^{18}$

\section{VI.2 Utilization, Labor, and Output at Instants of Investment}

We showed in section III that when the state variable $y \equiv Z / K$ reaches the trigger value $b$, the firm buys capital. At that instant, the capital stock jumps upward while $Z$ is fixed, so the state variable $y$ jumps downward. More precisely, the state variable $y$ jumps downward from the trigger value $b$ to the target point $c$. Thus, given the value of $Z$, we can infer that the capital stock jumps upward from $Z / b$ to $Z / c$. Equivalently, the capital 
stock immediately after investment is $b / c$ times as large as the capital stock immediately before investment. For small fixed cost $F$, Lemma 2 implies that the capital stock immediately after investment is approximately $1+2\left(\frac{15}{\alpha_{P} \gamma} \frac{F}{p} \phi\right)^{1 / 3}$ times as large as the capital stock immediately before investment.

When the capital stock jumps upward, the firm changes factor utilization and labor by discrete amounts. Let $\varepsilon_{\mu, K}$ be the elasticity of optimal factor utilization with respect to the capital stock, and let $\varepsilon_{L, K}$ be the elasticity of optimal employment by the firm with respect to the capital stock. Inspection of equations (12) and (13) reveals that

$$
\begin{gathered}
\varepsilon_{u, K}=-\frac{1-\theta}{\rho}<0, \text { and } \\
\varepsilon_{L, K}=1-\frac{1-\theta}{\rho}(\rho-\eta)=\frac{\theta \rho+(1-\theta) \eta}{\rho}>0 .
\end{gathered}
$$

Equations (40) and (41) indicate that at the instant the firm purchases capital, the rate of factor utilization $u$ jumps downward, and the level of employment $L$ jumps upward.

Since both employment $L$ and the capital stock $K$ jump upward, the labor-capital ratio, $L / K$ can rise, fall, or stay the same. If $\rho=\eta, \varepsilon_{L, K}=1$ so the labor-capital ratio is unchanged. If $\rho>\eta$, the fall in utilization reduces the unit operating cost of capital by more than it reduces the unit operating cost of labor. Therefore, the mix of factors shifts toward capital, and the labor-capital ratio falls. However, if $\rho<\eta$, the fall in utilization reduces the unit operating cost of labor by more than it reduces the unit operating cost of capital, so the labor-capital ratio increases. 
We can calculate the elasticity of output with respect to the capital stock at an instant of investment by using equations (40) and (41) along with the production function in equation (2) to obtain

$$
\varepsilon_{Q . K}=\frac{1}{\rho}[\theta(v-\alpha \eta+\alpha \rho)+(\alpha \eta+\beta \rho-v)]>0 .
$$

Thus, the purchase of capital leads to a jump upward in the firm's output. When the firm's output jumps upward the price of its output, $P$, jumps downward because the firm faces the downward-sloping demand curve in equation (1). It is worth noting that the price level jumps even though there are no menu costs or other costs of changing prices in the model. Indeed, in the short run, when the firm does not purchase capital, the output price evolves as a diffusion process and thus continuously changes.

\section{VI.3 Capacity Utilization as a Measure of the State Variable $y$}

The state variable $y$, which is the ratio of $Z$ to $K$, determines the timing of capital purchases, but is difficult to measure because both $Z$ and $K$ are difficult to measure. Recall that $Z$ is a geometrically weighted product of the demand shock $X_{1}$, the productivity shock $X_{2}$, the standardized unit labor cost $w$, and the standardized unit operating cost of capital $m$. With the possible exception of $w$ (which might be measured by the straight time wage rate), the components of $Z$ are difficult to measure. Also the capital stock, which is the denominator of $y$, is difficult to measure. Perhaps the component whose measurement has received the most attention is the productivity shock $X_{2}$. Early contributions to the literature on real business cycles measured productivity by the Solow residual. However, recent contributions have emphasized that the Solow residual includes variations in utilization as well as in productivity. ${ }^{19}$ 
Rather than measuring $Z$ and $K$ directly, $y$ can also be ascertained by observing Tobin's, or average, $q$. From its definition and using equation (20), we can write Tobin's $q$ as $V(Z, K) /(p K)=v(y) / p$. It is straightforward to verify that $v^{\prime}(y)>0$, so that Tobin's $q$ is a monotonic (increasing) function of $y$ and thus a sufficient statistic for the investment state variable, $y .{ }^{20,21}$ While this result shows that Tobin's $q$ remains a robust measure of the incentive to invest even with irreversibility and fixed costs, the potential measurement issues associated with both the numerator (asset values) and the denominator (the replacement value of the capital stock) also remain. ${ }^{22}$

Interestingly, our analysis shows that $m u^{\rho}$ is also a sufficient statistic for $y$. If this operating cost is interpreted as utilities, then Burnside, Eichenbaum, and Rebelo's (1995) use of electricity expenditures as a measure of capital services is precisely what our model would suggest. Moreover, the model suggests that electricity expenditures could be used directly as a measure of the investment state variable. This finding should be interpreted with some caution, however, because the result that $m u^{\rho}$ is a sufficient statistic for $y$ depends on the Cobb-Douglas formulation.

Because of these difficulties in measuring $y$, we present an alternative strategy based on capacity utilization. Here we distinguish capacity utilization from factor utilization represented by $u$ in the production function. Let the function $Q\left(K, X_{1}, X_{2}, w\right.$, $m$ ) represent the optimal amount of output given the capital stock $K$, the demand shock $X_{1}$, the productivity shock $X_{2}$, and the values of the standardized operating costs $w$ and $m$. Define capacity output $Q^{*} \equiv \sup _{X_{1}} Q\left(K, X_{1}, X_{2}, w, m\right)$ as the largest amount of output that the firm would produce with its current capital stock given the values of $X_{2}, w$, and $m$. When the firm is producing less than its capacity output, a small increase in the demand 
shock $X_{1}$ (which increases $X$ for a given value of $X_{2}$ ) would cause the firm to increase its output. However, if the demand shock is large enough, the firm will increase its capital stock. Define $X^{*}$ to be the smallest value of $X$ that induces the firm to buy capital, given $K, X_{2}, w$, and $m$. Capacity output $Q^{*}$ is the amount of output the firm would produce with its current capital stock and current values of $X_{2}, w$, and $m$, if $X$ were equal to $X^{*}$.

We showed in section III that it is optimal to purchase capital when $y \equiv Z / K=b$. Thus, given the capital stock $K$, the value of $Z$ that triggers investment is $Z^{*}=b K$. Using the definition of $Z$ in equation (15), the definition of the ratio $y$, and the fact that $Z^{*}=b K$ we obtain

$$
\frac{X}{X^{*}}=\left(\frac{Z}{Z^{*}}\right)^{\frac{(1-\theta) \Delta}{\rho}}=\left(\frac{y}{b}\right)^{\frac{(1-\theta) \Delta}{\rho}}
$$

Now use the expressions for optimal factor utilization and optimal employment in equations (12) and (13) (and define $u^{*}$ and $L^{*}$ as the optimal values of $u$ and $L$ at current factor prices and current capital stock when $X=X^{*}$ ) to obtain

$$
\begin{aligned}
& \frac{u}{u^{*}}=\left(\frac{X}{X^{*}}\right)^{\frac{1}{\Delta}} \\
& \frac{L}{L^{*}}=\left(\frac{X}{X^{*}}\right)^{\frac{\rho-\eta}{\Delta}}
\end{aligned}
$$

Because $X$ is less than or equal to $X^{*}$, equation (44) shows that factor utilization $u$ is less than or equal to $u^{*}$. However, according to equation (45), optimal $L$ will be less than, equal to, or greater than $L^{*}$ depending on whether $\rho$ is greater than, equal to, or less 
than $\eta$. Substituting equations (44) and (45) into the production function (2) (and recalling that productivity $X_{2}$ is taken as given) yields

$$
\frac{Q}{Q^{*}}=\left(\frac{u}{u^{*}}\right)^{v}\left(\frac{L}{L^{*}}\right)^{\alpha}=\left(\frac{X}{X^{*}}\right)^{\frac{v+\alpha(\rho-\eta)}{\Delta}}
$$

Finally substitute equation (43) into equation (46) to obtain

$$
\frac{Q}{Q^{*}}=\left(\frac{y}{b}\right)^{\frac{v+\alpha(\rho-\eta)}{\rho}(1-\theta)}
$$

Equation (47) shows the (increasing) monotonic relationship between capacity utilization $Q / Q^{*}$ and the level of the state variable $y$. Thus, we can use capacity utilization to measure the state variable $y$ without having to use measures of the demand shock $X_{1}$, the productivity shock $X_{2}$, the standardized operating costs $w$ and $m$, or the capital stock $K$. Of course, this measure requires data on capacity utilization, which itself may be subject to error.

\section{Conclusions}

We have derived the optimal investment behavior of a firm facing irreversibility and fixed costs of investment, and we have shown how this behavior affects the utilization and mix of factors employed by the firm. Since capital is quasi-fixed between instants of investment, both factor utilization and the labor-capital ratio may change in response to changes in demand and productivity, as well as to changes in unit operating costs. When investment occurs, the capital stock increases discretely, changing the 
returns to other factors and causing a discrete reduction in factor utilization and a discrete increase in employment.

These results suggest caution in interpreting factor dynamics as evidence of various forms of adjustment costs or market institutions. Here, employment exhibits high frequency small changes (in the "range of inaction") as well as lower frequency discrete increases, but there are no adjustment costs associated with labor directly. Thus, ignoring other quasi-fixed factors (here, capital) would produce apparently puzzling employment dynamics. Moreover, the level of employment may be unresponsive to changes in demand or productivity, yet there are no hiring and firing costs, implicit contracts, or other institutions usually associated with labor hoarding.

We have considered an extreme form of investment fixed costs, resulting in instants of investment separated by intervals of zero investment. Since most measures of capital expenditure are serially correlated, our model requires richer investment dynamics in order to match these data. The introduction of convex adjustment costs, stages-ofcompletion (as in Kydland and Prescott (1982)), or aggregation over capital types, for example, would not only generate serial correlation when investment is positive, but also "spread out" the labor and factor utilization adjustment associated with an increase in the capital stock. 


\section{Appendix A: Derivatives of $J^{1}(b, c)$ and $J^{2}(b, c)$}

Now evaluate the derivatives at $F=0$ and $b=c=\phi$ where $h \phi^{\gamma}=(r+\delta)\left(1-\frac{\gamma}{\alpha_{N}}\right) p$.

Notice that

$$
\left(1-\frac{\gamma}{\alpha_{P}}\right) h \phi^{\gamma}=(r+\delta)\left(1-\frac{\gamma}{\alpha_{N}}\right)\left(1-\frac{\gamma}{\alpha_{P}}\right) p=\rho(\gamma) p
$$

Therefore

$$
p=\left(1-\frac{\gamma}{\alpha_{P}}\right) \frac{h}{\rho(\gamma)} \phi^{\gamma}=\left(1-\frac{\gamma}{\alpha_{p}}\right) H \phi^{\gamma}
$$

The following relationships are useful:

$$
\begin{gathered}
p-H \phi^{\gamma}=-\frac{\gamma}{\alpha_{P}} H \phi^{\gamma} \\
\frac{\alpha_{P}}{\alpha_{P}-1} p=\frac{\alpha_{P}-\gamma}{\alpha_{P}-1} H \phi^{\gamma}
\end{gathered}
$$

$$
\begin{aligned}
& J^{1}{ }_{b}(b, c)=\alpha_{P}\left(p-H c^{\gamma}\right) b^{\alpha_{p}-1}+\gamma H b^{\gamma-1} c^{\alpha_{p}} \\
& J^{1}{ }_{b}(\phi, \phi)=-\gamma H \phi^{\gamma} \phi^{\alpha_{P}-1}+\gamma H \phi^{\gamma-1} \phi^{\alpha_{P}}=0 \\
& J^{1}{ }_{c}(b, c)=-\gamma H c^{\gamma-1} b^{\alpha_{P}}-\alpha_{P}\left(p-H b^{\gamma}\right) c^{\alpha_{P}-1} \\
& J^{1}{ }_{c}(\phi, \phi)=-\gamma H \phi^{\gamma-1} \phi^{\alpha_{P}}+\gamma H \phi^{\gamma} \phi^{\alpha_{r}-1}=0 \\
& J^{1}{ }_{b b}(b, c)=\alpha_{P}\left(\alpha_{P}-1\right)\left(p-H c^{\gamma}\right) b^{\alpha_{P}-2}+\gamma(\gamma-1) H b^{\gamma-2} c^{\alpha_{p}} \\
& J^{1}{ }_{b b}(\phi, \phi)=-\gamma\left(\alpha_{P}-1\right) H \phi^{\gamma} \phi^{\alpha_{p}-2}+\gamma(\gamma-1) H \phi^{\gamma-2} \phi^{\alpha_{P}}=\left(\gamma-\alpha_{P}\right) \gamma H \phi^{\gamma+\alpha_{P}-2} \\
& J^{\prime}{ }_{b c}(b, c)=-\gamma \alpha_{P} H c^{\gamma-1} b^{\alpha_{P}-1}+\alpha_{P} \gamma H b^{\gamma-1} c^{\alpha_{p}-1} \\
& J^{1}{ }_{b c}(\phi, \phi)=-\gamma \alpha_{P} H \phi^{\gamma+\alpha_{P}-2}+\alpha_{P} \gamma H \phi^{\gamma+\alpha_{P}-2}=0 \\
& J^{1}{ }_{c c}(b, c)=-\gamma(\gamma-1) H c^{\gamma-2} b^{\alpha_{p}}-\alpha_{P}\left(\alpha_{P}-1\right)\left(p-H b^{\gamma}\right) c^{\alpha_{p}-2} \\
& J^{1}{ }_{c c}(\phi, \phi)=-\gamma(\gamma-1) H \phi^{\gamma+\alpha_{P}-2}+\gamma\left(\alpha_{P}-1\right) H \phi^{\gamma+\alpha_{P}-2}=-\left(\gamma-\alpha_{P}\right) \gamma H \phi^{\gamma+\alpha_{P}-2} \\
& J^{1}{ }_{b b b}(b, c)=\alpha_{P}\left(\alpha_{p}-1\right)\left(\alpha_{p}-2\right)\left(p-H c^{\gamma}\right) b^{\alpha_{P}-3}+\gamma(\gamma-1)(\gamma-2) H b^{\gamma-3} c^{\alpha_{P}} \\
& J^{1}{ }_{b b b}(\phi, \phi)=-\gamma\left(\alpha_{P}-1\right)\left(\alpha_{P}-2\right) H \phi^{\gamma+\alpha_{P}-3}+\gamma(\gamma-1)(\gamma-2) H \phi^{\gamma+\alpha_{P}-3} \\
& =\gamma H \phi^{\gamma+\alpha_{P}-3}\left[-\left(\alpha_{P}-1\right)\left(\alpha_{P}-2\right)+(\gamma-1)(\gamma-2)\right] \\
& =\gamma H \phi^{\gamma+\alpha_{P}-3}\left[-\alpha_{P}^{2}+3 \alpha_{P}+\gamma^{2}-3 \gamma\right]=\gamma H \phi^{\gamma+\alpha_{P}-3}\left[\left(\gamma-\alpha_{P}\right)\left(\gamma+\alpha_{P}\right)-3\left(\gamma-\alpha_{P}\right)\right] \\
& =\left(\gamma-\alpha_{P}\right)\left(\gamma+\alpha_{p}-3\right) \gamma H \phi^{\gamma+\alpha_{p}-3} \\
& J_{b b c}^{1}(b, c)=-\gamma \alpha_{P}\left(\alpha_{P}-1\right) H c^{\gamma-1} b^{\alpha_{p}-2}+\alpha_{P} \gamma(\gamma-1) H b^{\gamma-2} c^{\alpha_{p}-1} \\
& J^{1}{ }_{b b c}(\phi, \phi)=-\gamma \alpha_{P}\left(\alpha_{P}-1\right) H \phi^{\gamma+\alpha_{P}-3}+\alpha_{P} \gamma(\gamma-1) H \phi^{\gamma+\alpha_{P}-3}=\alpha_{P} \gamma\left(\gamma-\alpha_{P}\right) H \phi^{\gamma+\alpha_{P}-3}
\end{aligned}
$$




$$
\begin{aligned}
& J^{1}{ }_{b c c}(b, c)=-\gamma(\gamma-1) \alpha_{P} H c^{\gamma-2} b^{\alpha_{p}-1}+\alpha_{P}\left(\alpha_{P}-1\right) \gamma H b^{\gamma-1} c^{\alpha_{p-2}} \\
& J_{b c c}^{1}(\phi, \phi)=-\gamma(\gamma-1) \alpha_{P} H \phi^{\gamma+\alpha_{p}-3}+\alpha_{P}\left(\alpha_{P}-1\right) \gamma H \phi^{\gamma+\alpha_{P}-3}=\gamma \alpha_{P} H \phi^{\gamma+\alpha_{p}-3}\left(\alpha_{P}-\gamma\right) \\
& J^{1}{ }_{c c c}(b, c)=-\gamma(\gamma-1)(\gamma-2) H c^{\gamma-3} b^{\alpha_{p}}-\alpha_{p}\left(\alpha_{p}-1\right)\left(\alpha_{P}-2\right)\left(p-H b^{\gamma}\right) c^{\alpha_{p}-3} \\
& J_{c c c}^{1}(\phi, \phi)=-\gamma(\gamma-1)(\gamma-2) H \phi^{\gamma+\alpha_{p}-3}+\gamma\left(\alpha_{p}-1\right)\left(\alpha_{P}-2\right) H \phi^{\gamma+\alpha_{p}-3} \\
& =\gamma H \phi^{\gamma+\alpha_{P}-3}\left[-\gamma^{2}+3 \gamma+\alpha_{P}{ }^{2}-3 \alpha_{P}\right] \\
& =\gamma H \phi^{\gamma+\alpha_{P}-3}\left[\left(\alpha_{P}-\gamma\right)\left(\alpha_{P}+\gamma\right)-3\left(\alpha_{P}-\gamma\right)\right] \\
& =\gamma\left(\alpha_{P}-\gamma\right) H \phi^{\gamma+\alpha_{p}-3}\left(\alpha_{P}+\gamma-3\right) \\
& J^{2}(b, c ; F) \equiv \frac{\alpha_{P}-\gamma}{(\gamma-1)\left(\alpha_{P}-1\right)} H\left(b^{\gamma-1}-c^{\gamma-1}\right)+\frac{\alpha_{P}-\gamma}{\alpha_{p}-1} H \phi^{\gamma}\left(b^{-1}-c^{-1}\right)-F \\
& =\frac{\alpha_{P}-\gamma}{\alpha_{P}-1} H\left[\frac{1}{\gamma-1}\left(b^{\gamma-1}-c^{\gamma-1}\right)+\phi^{\gamma}\left(b^{-1}-c^{-1}\right)\right]-F \\
& J^{2}(b, c ; F)=\frac{\alpha_{P}-\gamma}{\alpha_{P}-1} H\left[\frac{1}{\gamma-1}\left(b^{\gamma-1}-c^{\gamma-1}\right)+\phi^{\gamma}\left(b^{-1}-c^{-1}\right)\right]-F \\
& J_{b}^{2}(b, c ; F)=\frac{\alpha_{P}-\gamma}{\alpha_{P}-1} H\left[b^{\gamma-2}-\phi^{\gamma} b^{-2}\right] \\
& J^{2}{ }_{b}(\phi, \phi ; 0)=0 \\
& J^{2}{ }_{c}(b, c ; F)=-\frac{\alpha_{P}-\gamma}{\alpha_{P}-1} H\left[c^{\gamma-2}-\phi^{\gamma} c^{-2}\right] \\
& J^{2}{ }_{c}(\phi, \phi ; 0)=0 \\
& J_{b b}^{2}(b, c ; F)=\frac{\alpha_{P}-\gamma}{\alpha_{P}-1} H\left[(\gamma-2) b^{\gamma-3}+2 \phi^{\gamma} b^{-3}\right] \\
& J^{2}{ }_{b b}(\phi, \phi ; 0)=\frac{\alpha_{P}-\gamma}{\alpha_{P}-1} \gamma H \phi^{\gamma-3} \\
& J^{2}{ }_{b c}(b, c ; F)=0 \\
& J^{2}{ }_{c c}(b, c ; F)=-\frac{\alpha_{P}-\gamma}{\alpha_{P}-1} H\left[(\gamma-2) c^{\gamma-3}+2 \phi^{\gamma} c^{-3}\right] \\
& J^{2}{ }^{2}(\phi, \phi ; 0)=-\frac{\alpha_{P}-\gamma}{\alpha_{P}-1} \gamma H \phi^{\gamma-3} \\
& J_{b b b}^{2}(b, c ; F)=\frac{\alpha_{P}-\gamma}{\alpha_{P}-1} H\left[(\gamma-2)(\gamma-3) b^{\gamma-4}-6 \phi^{\gamma} b^{-4}\right] \\
& J_{b b b}^{2}(\phi, \phi ; 0)=\frac{\alpha_{P}-\gamma}{\alpha_{P}-1} H \phi^{\gamma-4}[(\gamma-2)(\gamma-3)-6]=\frac{\alpha_{P}-\gamma}{\alpha_{P}-1} \gamma(\gamma-5) H \phi^{\gamma-4} \\
& J^{2}{ }_{b b c}(b, c ; F)=0 \\
& J^{2}{ }_{b c c}(b, c ; F)=0
\end{aligned}
$$




$$
\begin{aligned}
& J_{c c c}^{2}(b, c ; F)=-\frac{\alpha_{P}-\gamma}{\alpha_{P}-1} H\left[(\gamma-2)(\gamma-3) c^{\gamma-4}-6 \phi^{\gamma} c^{-4}\right] \\
& J_{c c c}^{2}(\phi, \phi ; 0)=-\frac{\alpha_{P}-\gamma}{\alpha_{P}-1} H \phi^{\gamma-4}[(\gamma-2)(\gamma-3)-6]=-\frac{\alpha_{P}-\gamma}{\alpha_{P}-1} \gamma(\gamma-5) H \phi^{\gamma-4}
\end{aligned}
$$

(ignoring $F$ ):

$$
\begin{aligned}
& J^{i}(b, c ; F)=J^{i}(\phi, \phi ; 0)+J^{i}{ }_{b}(\phi, \phi ; 0) \tilde{b}+J^{i}{ }_{c}(\phi, \phi ; 0) \tilde{c} \\
& +\frac{1}{2} J_{b b}^{i}(\phi, \phi ; 0) \tilde{b}^{2}+J_{b c}^{i}(\phi, \phi ; 0) \tilde{b} \widetilde{c}+\frac{1}{2} J^{i}{ }_{c c}(\phi, \phi ; 0) \tilde{c}^{2} \\
& +\frac{1}{6} J_{b b b}^{i}(\phi, \phi ; 0) \tilde{b}^{3}+\frac{1}{2} J_{b b c}^{i}(\phi, \phi ; 0) \tilde{b}^{2} \tilde{c}+\frac{1}{2} J_{b c c}^{i}(\phi, \phi ; 0) \tilde{b} \tilde{c}^{2}+\frac{1}{6} J^{i}{ }_{c c c}(\phi, \phi ; 0) \tilde{c}^{3} \\
& J^{1}(b, c ; F)=\frac{1}{2}\left(\gamma-\alpha_{P}\right) \gamma H \phi^{\gamma+\alpha_{p}-2}\left(\tilde{b}^{2}-\tilde{c}^{2}\right)+\frac{1}{6}\left(\gamma-\alpha_{P}\right)\left(\gamma+\alpha_{P}-3\right) \gamma H \phi^{\gamma+\alpha_{\rho}-3}\left(\tilde{b}^{3}-\tilde{c}^{3}\right) \\
& +\frac{1}{2} \alpha_{p} \gamma\left(\gamma-\alpha_{p}\right) H \phi^{\gamma+\alpha_{p}-3}\left(\tilde{b}^{2} \tilde{c}-\tilde{b} \widetilde{c}^{2}\right) \\
& J^{2}(b, c ; F)=\frac{\alpha_{p}-\gamma}{\alpha_{p}-1} \gamma H \phi^{\gamma-4}\left[\frac{1}{2} \phi\left(\tilde{b}^{2}-\tilde{c}^{2}\right)+\frac{1}{6}(\gamma-5)\left(\tilde{b}^{3}-\tilde{c}^{3}\right)\right]-F
\end{aligned}
$$




\section{Appendix B: Properties of the roots of $v(\eta)=0$}

Recall that $v(\eta) \equiv-\frac{1}{2} \sigma^{2} \eta^{2}-\left(\mu-\frac{1}{2} \sigma^{2}\right) \eta+r+\delta=0$. Note that $\nu(\eta)$ is strictly concave, $v(0)=r+\delta>0$, and $v(1)=r-\mu>0$ so that $v(\gamma)>0$. Also, note that $v(\eta)=0$ has two distinct roots, $\alpha_{P}>0$ and $\alpha_{N}<0$, which satisfy $\alpha_{N}<0<\gamma<1<\alpha_{P}$. Differentiating $\cup(\eta)$ with respect to $\eta$ and evaluating the derivative at the roots yields

$$
v^{\prime}\left(\alpha_{i}\right)=-\sigma^{2} \alpha_{i}-\left(\mu-\frac{1}{2} \sigma^{2}\right) \quad i=N, P
$$

The concavity of $v(\eta)$ implies that $v^{\prime}\left(\alpha_{N}\right)>0$ and $v^{\prime}\left(\alpha_{P}\right)<0$. Symmetry implies that $v^{\prime}\left(\alpha_{P}\right)=-v^{\prime}\left(\alpha_{N}\right)$.

Totally differentiating $\nu(\eta)$ with respect to $\eta, \mu, \sigma^{2}$, and $r$, and evaluating the derivatives at $\eta=\alpha_{i}$ yields

$$
v^{\prime}\left(\alpha_{i}\right) d \alpha_{i}-\alpha_{i} d \mu-\frac{1}{2} \alpha_{i}\left(\alpha_{i}-1\right) d \sigma^{2}+d r=0 . \quad i=N, P
$$

Equation (B.2) implies

$$
\begin{gathered}
\frac{d \alpha_{i}}{d \mu}=\frac{\alpha_{i}}{v^{\prime}\left(\alpha_{i}\right)}<0 \quad i=N, P \\
\frac{d \alpha_{i}}{d r}=-\frac{1}{v^{\prime}\left(\alpha_{i}\right)} \quad i=N, P
\end{gathered}
$$

and

$$
\frac{d \alpha_{i}}{d \sigma^{2}}=\frac{1}{2} \frac{\alpha_{i}\left(\alpha_{i}-1\right)}{v^{\prime}\left(\alpha_{i}\right)} . \quad i=N, P
$$




\section{Appendix C: Approximate values of $\tilde{b}$ and $\tilde{c}$}

Use the derivatives in Appendix A to take a third-order expansion of $J^{1}(b, c)$ and set the expansion equal to zero to obtain

$$
\frac{1}{2} \phi\left(\tilde{b}^{2}-\tilde{c}^{2}\right) \cong-\frac{1}{6}\left(\gamma+\alpha_{P}-3\right)\left(\tilde{b}^{3}-\tilde{c}^{3}\right)-\frac{1}{2} \alpha_{P}\left(\tilde{b}^{2} \tilde{c}-\tilde{b} \tilde{c}^{2}\right)
$$

Taking a third-order expansion of $J^{2}(b, c ; F)$ and setting the expansion equal to zero yields

$$
\frac{\alpha_{P}-\gamma}{\alpha_{P}-1} \gamma H \phi^{\gamma-4}\left[\frac{1}{2} \phi\left(\tilde{b}^{2}-\tilde{c}^{2}\right)+\frac{1}{6}(\gamma-5)\left(\tilde{b}^{3}-\tilde{c}^{3}\right)\right] \cong F
$$

Substituting the right hand side of equation (C.1) for $\frac{1}{2} \phi\left(\tilde{b}^{2}-\tilde{c}^{2}\right)$ in equation (C.2) and rearranging terms yields

$$
-\frac{1}{6} \frac{\alpha_{P}-\gamma}{\alpha_{P}-1} \gamma H \phi^{\gamma-4}(\tilde{b}-\tilde{c})\left[2\left(\alpha_{P}-1\right) \tilde{b} \tilde{c}+\left(\alpha_{P}+2\right)(\tilde{b}+\tilde{c})^{2}\right] \cong F
$$

For small $F, \tilde{c} \cong-\tilde{b}$. More precisely, $\tilde{b} \tilde{c}$ is the order of $\tilde{b}^{2}$ and $(\tilde{b}+\tilde{c})^{2}$ is of the order of $\tilde{b}^{4}$ as we show at the end of this appendix. Ignoring the term with $(\tilde{b}+\tilde{c})^{2}$ and setting $\widetilde{c} \cong-\tilde{b}$ yields

$$
\tilde{b}^{3} \cong \frac{1.5 F}{\left(\alpha_{p}-\gamma\right) \gamma H \phi^{\gamma}} \phi^{4}
$$

Multiply both sides of equation (34) by $\frac{1}{v(\gamma)}\left(1-\frac{\gamma}{\alpha_{P}}\right)$ and use equation (24) and the definition $H \equiv \frac{h}{v(\gamma)}$ to obtain

$$
\left(1-\frac{\gamma}{\alpha_{p}}\right) H \phi^{\gamma}=p
$$

Finally, substitute equation (C.5) into equation (C.4) to obtain

$$
\tilde{b} \cong\left(\frac{1.5}{\alpha_{P} \gamma} \frac{F}{p} \phi\right)^{1 / 3} \phi
$$


Next, we derive the target point $c$ as a function of the trigger point $b$. Divide both sides of equation (C.1) by $(\tilde{b}-\tilde{c})$ and rearrange to obtain

$$
3 \phi(\tilde{b}+\tilde{c}) \cong-\left(\gamma+\alpha_{P}-3\right)(\tilde{b}+\tilde{c})^{2}+\left(\gamma-3-2 \alpha_{P}\right) \tilde{b} \tilde{c}
$$

We show below that $(\tilde{b}+\tilde{c})^{2}$ is of the order of $\tilde{b}^{4}$. Ignoring this term in equation (C.7) yields

$$
\widetilde{c} \cong-\frac{3 \phi}{3 \phi-\left(\gamma-3-2 \alpha_{P}\right) \tilde{b}} \tilde{b}
$$

Observe that for small values of $\tilde{b}, \tilde{b} \tilde{c}=-\frac{3 \phi}{3 \phi-\left(\gamma-2 \alpha_{p}-3\right) \tilde{b}} \tilde{b}^{2}$ is of the order of $\tilde{b}^{2}$, $\tilde{b}+\tilde{c}=\frac{3-\gamma+2 \alpha_{P}}{3 \phi-\left(\gamma-2 \alpha_{P}-3\right) \tilde{b}} \tilde{b}^{2}$ is of the order of $\tilde{b}^{2}$, and $(\tilde{b}+\tilde{c})^{2}=\left[\frac{\gamma-2 \alpha_{p}-3}{3 \phi-\left(\gamma-2 \alpha_{p}-3\right) \tilde{b}}\right]^{2} \tilde{b}^{4}$ is of the order of $\tilde{b}^{4}$. 


\section{Appendix D: Proofs of Lemmas and Propositions}

Proof of Lemma 1: Define $w \equiv \ln y$. Use Ito's Lemma to obtain $d w=\left(\mu_{y}-(1 / 2) \sigma_{y}{ }^{2}\right) d t+$ $\sigma_{y} d z$. The distribution of first passage times of $y$ from $y_{0}$ to $y_{1}$ is identical to the distribution of first passages times of $w$ from $\ln y_{0}$ to $\ln y_{1}$. The expected value of the first passage time of $w$ from $\ln y_{0}$ to $\ln y_{1}$ is $\frac{\ln \left(y_{1} / y_{0}\right)}{\mu_{y}-(1 / 2) \sigma_{y}{ }^{2}}$ (Cox and Miller, p. 222). q.e.d.

Proof of Lemma 2: If $F$ is small, $\ln (b / c)=\ln b-\ln c=\ln (\phi+\tilde{b})-\ln (\phi+\tilde{c})=$ $\ln (1+\tilde{b} / \phi)-\ln (1+\tilde{c} / \phi) \cong(\tilde{b}-\tilde{c}) / \phi \cong 2 \tilde{b} / \phi$. Equation (36a) implies that $\frac{2 \tilde{b}}{\phi} \cong 2\left(\frac{1.5}{\alpha_{P} \gamma} \frac{F}{p} \phi\right)^{1 / 3} \cdot$ q.e.d.

Proof of Proposition 1: Inspect Lemmas 1 and 2 and Corollary 1 to Lemma 1. q.e.d.

Proof of Proposition 2: Lemma 2 implies that $\frac{d \ln \left(\frac{b}{c}\right)}{d \sigma_{y}{ }^{2}}$ has the same sign as $\frac{d\left(\frac{\phi}{\alpha_{p}}\right)}{d \sigma_{y}{ }^{2}}$.

Differentiate to obtain $\frac{d\left(\phi / \alpha_{P}\right)}{d \sigma_{y}{ }^{2}}=\frac{1}{\alpha_{P}} \frac{d \phi}{d \sigma_{y}{ }^{2}}-\frac{\phi}{\alpha_{P}{ }^{2}} \frac{d \alpha_{P}}{d \sigma_{y}{ }^{2}}=\frac{1}{\alpha_{P}} \frac{d \phi}{d \alpha_{N}} \frac{d \alpha_{N}}{d \sigma_{y}^{2}}-\frac{\phi}{\alpha_{P}^{2}} \frac{d \alpha_{P}}{d \sigma_{y}^{2}}$.

Calculate $\frac{d \phi}{d \alpha_{N}}$ by totally differentiating equation (34) with respect to $\phi$ and $\alpha_{N}$ and simplifying to obtain $\frac{d \phi}{d \alpha_{N}}=\frac{(r+\delta) p}{h \phi^{\gamma}} \frac{1}{\alpha_{N}{ }^{2}} \phi$. Use equation (34) to obtain $\frac{d \phi}{d \alpha_{N}}=\frac{\phi}{\alpha_{N}\left(\alpha_{N}-\gamma\right)}>0$. Therefore, $\frac{d\left(\frac{\phi}{\alpha_{P}}\right)}{d \sigma_{y}^{2}}=\frac{\phi}{\alpha_{P} \alpha_{N}\left(\alpha_{N}-\gamma\right)} \frac{d \alpha_{N}}{d \sigma_{y}^{2}}-\frac{\phi}{\alpha_{P}^{2}} \frac{d \alpha_{P}}{d \sigma_{y}^{2}}$. Use equation (B.15) to obtain $\frac{d\left(\frac{\phi}{\alpha_{P}}\right)}{d \sigma_{y}^{2}}=\frac{1}{2} \frac{\phi}{\alpha_{P}}\left[\frac{1}{\left(\alpha_{N}-\gamma\right)} \frac{\left(\alpha_{N}-1\right)}{v^{\prime}\left(\alpha_{N}\right)}-\frac{\left(\alpha_{P}-1\right)}{v^{\prime}\left(\alpha_{P}\right)}\right]$. Use the fact that $v^{\prime}\left(\alpha_{N}\right)=-v^{\prime}\left(\alpha_{P}\right)$ to obtain $\frac{d\left(\phi / \alpha_{P}\right)}{d \sigma_{y}^{2}}=\frac{1}{2} \frac{\phi}{\alpha_{P}} \frac{1}{v^{\prime}\left(\alpha_{N}\right)}\left[\frac{\alpha_{N}-1}{\alpha_{N}-\gamma}+\alpha_{P}-1\right]>0$. q.e.d.

Proof of Proposition 3: According to Proposition 1, $E\{T(c, b)\}$ can be written as a product of two factors: (1) $\ln (b / c)$ and (2) $\frac{1}{\mu_{y}-(1 / 2) \sigma_{y}{ }^{2}}$. An increase in $\sigma_{y}{ }^{2}$ increases the 
first factor if $F$ is small (Proposition 2) and increases the second factor (Corollary 2 to Lemma 1). q.e.d.

Proof of Proposition 4: Define $w \equiv \ln y$. Use Ito's Lemma to obtain $d w=\left(\mu_{y}-(1 / 2) \sigma_{y}^{2}\right) d t$ $+\sigma_{y} d z$. The distribution of first passage times of $y$ from $y_{0}$ to $y_{1}$ is identical to the distribution of first passages times of $w$ from $\ln y_{0}$ to $\ln y_{1}$. Let $g(t)$ be the density function of first passage times of $w$ from $\ln y_{0}$ to $\ln y_{1}$. The moment generating function of this distribution is $E\left\{e^{-s T\left(y_{0}, y_{1}\right)}\right\}=\int_{0}^{\infty} e^{-s t} g(t) d t=\exp \left[-\left(\ln y_{1}-\ln y_{0}\right) \lambda_{2}(s)\right]$ (Cox and Miller, p. 221, eq. (75)) where $\lambda_{2}(s)$ is the positive root of the quadratic equation $A(\lambda) \equiv \frac{1}{2} \sigma_{y}{ }^{2} \lambda^{2}+\left(\mu_{y}-\frac{1}{2} \sigma_{y}{ }^{2}\right) \lambda-s=0$. If $s=r+\delta$, the roots of $A(\lambda)=0$ are identical to the roots of $U(\eta)=0$, so that $\lambda_{2}=\alpha_{P}>1$ and $E\left\{e^{-(r+\delta) r\left(y_{0}, y_{1}\right)}\right\}=\exp \left[-\left(\ln y_{1}-\ln y_{0}\right) \alpha_{P}\right]$. Differentiating this equation and using equation (5) yields

$$
\frac{d E\left\{e^{-(r+\delta) T\left(y_{0}, y_{1}\right)}\right\}}{d \sigma_{y}{ }^{2}}=-\frac{1}{2} \frac{\alpha_{P}\left(\alpha_{P}-1\right)}{\rho^{\prime}\left(\alpha_{P}\right)}\left(\ln y_{1}-\ln y_{0}\right) E\left\{e^{-(r+\delta) T\left(y_{0}, y_{1}\right)}\right\}>0 \text {. q.e.d. }
$$




\section{References}

Abel, A. B. and Eberly, J.C.

(1995) The Effects of Irreversibility and Uncertainty on Capital Accumulation, National Bureau of Economic Research Working Paper 5363. and

(1996a) Investment and q with Fixed Costs: An Empirical Analysis, manuscript, the Wharton School of the University of Pennsylvania. and

(1996b) Optimal Investment with Costly Reversibility. Review of Economic Studies, 63: $581-93$.

Bertola, G.

(1988) Adjustment Costs and Dynamic Factor Demands: Investment and Employment Under Uncertainty (Doctoral dissertation, Massachusetts Institute of Technology).

Bentolila, S. and Bertola,G.

(1990) Firing Costs and Labor Demand: How Bad is Eurosclerosis? Review of Economic Studies, 57: 381-402.

Bernanke, B. S.

(1983) Irreversibility, Uncertainty, and Cyclical Investment. Quarterly Journal of Economics, 98: 85-106. 
Burnside, C., Eichenbaum, M. and Rebelo, S.

(1993) Labor Hoarding and the Business Cycle. Journal of Political Economy, 101: $245-73$. and

(1995) Capacity Utilization and Returns to Scale. NBER Macroeconomics Annual 1995, eds. B. S. Bernanke and J. J. Rotemberg. Cambridge, MA: The MIT Press, 67-110.

Caballero, R.J., Engel, E.M.R.A., Haltiwanger, J.C.

(1995) Plant-Level Adjustment and Aggregate Investment Dynamics. Brookings Papers on Economic Activity, 2:1995, 1-39. and Leahy, J.V.

(1996) Fixed Costs: The Demise of Marginal q. National Bureau of Economic Research Working Paper No. 5508.

Cox, D. R., and Miller, H.D.

(1980) The Theory of Stochastic Processes, New York: Chapman and Hall.

Delgado, F. and Dumas, B.

(1994) How Far Apart Can Two Riskless Interest Rates Be? (One Moves, the Other One Does Not), Rodney L. White Center for Financial Research, The Wharton School of the University of Pennsylvania, Working Paper No. 1894. 
Dixit, A.

(1991) Analytical Approximations in Models of Hysteresis. Review of Economic Studies, 58 (1991), 141-151.

and Robert S. Pindyck, R.S.

(1994) Investment Under Uncertainty. Princeton, NJ: Princeton University Press.

Eberly, J. C.,

(1997) International Evidence on Investment and Fundamentals. European Economic Review, forthcoming. and van Mieghem, J.

(1997) Multi-factor Dynamic Investment under Uncertainty. Journal of Economic Theory. forthcoming.

Fay, J. A. and Medoff, J.L.

(1985) Labor and Output Over the Business Cycle: Some Direct Evidence. American Economic Review, 75: 638-55.

Greenwood, J., Hercowitz, Z., and Huffman, G.W.

(1988) Investment, Capacity Utilization, and the Real Business Cycle. American Economic Review, 78: 402-17.

Hopenhayn, H. and Rogerson, R. 
(1993) Job Turnover and Policy Evaluation: A General Equilibrium Analysis. Journal of Political Economy, 101: 915-38.

Jorgenson, D. W.

(1963) Capital Theory and Investment Behavior. American Economic Review Papers and Proceedings, 53: 247-59.

Kydland, F. E. and Prescott, E.C.

(1988) The Workweek of Capital and Its Cyclical Implications. Journal of Monetary Economics, 21: 343-60. and

(1982) Time to Build and Aggregate Fluctuations," Econometrica, 50: 1345-70.

McDonald, R. and Siegel, D.R.

(1986) The Value of Waiting to Invest. Quarterly Journal of Economics, 101: 70728.

Pindyck, R. S.

(1988) Irreversible Investment, Capacity Choice, and the Value of the Firm. American Economic Review, 78: 969-85.

Shreve, S.E. and Soner, H.M.

(1994) Optimal Investment and Consumption with Transaction Costs. Annals of Applied Probability, 4: 609-92. 
* We thank Susanto Basu, Marcelo Veracierto, the Penn Macro Lunch Group, and seminar participants at the Federal Reserve Banks of Minneapolis, New York, and St. Louis, and the Board of Governors of the Federal Reserve System for helpful comments.

${ }^{1}$ Homotheticity implies that ratios of marginal products depend only on ratios of factors. ${ }^{2}$ Bernanke (1983), McDonald and Siegel (1986), Bertola (1988) and Pindyck (1988) present early work focusing on the investment decision with irreversibility and uncertainty. Additional work in this area and the general principles are covered in Dixit and Pindyck (1994).

${ }^{3}$ Eberly and van Mieghem (1997) characterize investment with costly reversibility of many factors, but do not allow for relative price variability or fixed costs.

${ }^{4}$ The assumption of a common utilization rate for capital and labor captures the notion that the workweek of capital and the workweek of labor are equal. Greenwood, Hercowitz, and Huffman (1988), Kydland and Prescott (1988), and Burnside, Eichenbaum, and Rebelo (1993) allow for endogenous utilization of only one factor, either capital or labor (effort). In later work, Burnside, Eichenbaum, and Rebelo (1995) allow for variable utilization of both capital and labor in their analytic structure, though they focus on capital utilization in their empirical work. Their approach abstracts from labor-hoarding behavior, and also ignores capital dynamics since the capital stock is taken as given. 
${ }^{5}$ The restriction $v \eta>\alpha$ is required for the first-order conditions in equations (7) and (8) to have a solution with positive values of revenue, capital, and utilization. To verify this statement substitute equation (8) into equation (7) to obtain $(v-\alpha \eta)\left(1-\frac{1}{\varepsilon}\right) R=\rho m u^{\rho} K$. ${ }^{6}$ These geometric Brownian motions can be correlated with each other and can also be correlated with the geometric Brownian motion for the revenue shock $X$.

${ }^{7}$ Note that $\Delta>\rho-v-\alpha \rho+\alpha \eta=(1-\alpha) \rho-v+\alpha \eta \geq \beta \rho-v+\alpha \eta \geq \beta+\alpha-v \geq 0$.

${ }^{8}$ The following relationship is useful in derivations $(1-\theta)_{\Delta}=\rho\left[1-\left(1-\frac{1}{\varepsilon}\right)(\alpha+\beta)\right]$.

${ }^{9}$ The exponent of $\omega$ in equation (13) is $1-(\rho-\eta) \frac{\alpha}{\alpha+\beta} \frac{1-\theta}{\rho}>1-\frac{\alpha}{\alpha+\beta}(1-\theta)>0$.

${ }^{10}$ Our assumption that the components of $Z$ follow geometric Brownian motions allows an analytic characterization of dynamic investment behavior. However, our analysis of utilization and employment in the short run-when the capital stock is fixed--does not depend on this assumption.

${ }^{11}$ We will show that the optimal policy is for the firm to invest whenever $Z_{t} / K_{t}$ reaches $b$, and to buy enough capital so that $Z_{t} / K_{t}$ equals $c<b$. Thus, the capital stock always satisfies $K_{t} \leq(1 / c) Z_{m, t}$ where $Z_{m, t}$ is the maximum value that $Z$ has reached up to and including time $t$. Because the profit at time $t$ is $\pi_{t}=A_{\pi} Z_{t}^{1-\theta} K_{t}^{\theta}$, we have $\pi_{t} \leq A_{\pi}(1 / c)^{\theta} Z_{m, t}$. It can be shown (Abel and Eberly, 1995, equation (11)) that $E\left\{\max _{0 \leq s \leq t} Z_{s} \mid Z_{0}=1\right\}=\frac{\mu_{z}+\frac{1}{2} \sigma_{z}^{2}}{\mu_{z}} \Phi\left(\frac{\mu_{z}+\frac{1}{2} \sigma_{z}^{2}}{\sigma_{z}} t^{1 / 2}\right) \exp \left[\mu_{z} t\right]+\frac{\mu_{z}-\frac{1}{2} \sigma_{z}^{2}}{\mu_{z}} \Phi\left(-\frac{\mu_{z}-\frac{1}{2} \sigma_{z}^{2}}{\sigma_{z}} t^{1 / 2}\right)$ 
Therefore, since $r>\mu_{\mathrm{Z}}, \lim _{s \rightarrow \infty} e^{-r(s-t)} E_{\mathrm{f}}\left\{\pi_{s}\right\}=0$, and hence the expected present value of expected profits over the infinite future is finite.

${ }^{12}$ Caballero and Leahy (1996) prevent the fixed cost of investment from becoming either trivial or overwhelming by specifying the fixed cost to be proportional to the existing capital stock. However, with their specification, current investment affects future fixed costs, and this effect must be taken into account in determining optimal investment. Our formulation avoids this complication while preventing fixed costs from becoming trivial or overwhelming.

${ }^{13}$ Lower bounds for the trigger and return points can be obtained by noting that $H b^{\gamma}>p$ and $H c^{\gamma}>p$ which imply $h b^{\gamma}>v(\gamma) p$ and $h c^{\gamma}>v(\gamma) p$, where $h b^{\gamma}$ and $h c^{\gamma}$ are the marginal profits of capital at the trigger and return points respectively.

${ }^{14}$ See, for example, Abel and Eberly (1996b).

${ }^{15}$ In models in a variety of economic contexts, Abel and Eberly (1996b), Delgado and Dumas (1994), Dixit (1991), and Shreve and Soner (1994) have found a similar locally cubic property.

${ }^{16} \mathrm{An}$ increase in $\sigma_{y}^{2}$ for a given $\mu_{y}$ is a mean-preserving spread of future values of $y$. However, it is not a mean-preserving spread for future $y^{\kappa}$ for $\kappa \neq 1$. Abel and Eberly (1995) analyze increases in $\sigma_{y}^{2}$ accompanied by a changes in $\mu_{y}$ that preserve the mean of future values of $y^{\kappa}$.

${ }^{17}$ If the decrease in $C / X$ were due at least partly to an increase in productivity, then the apparent returns to labor would be even greater than calculated in equation (39). 
${ }^{18}$ Moreover, these doubts are strengthened by the finding in the next subsection that employment also jumps when the capital stock jumps, even though there are no fixed costs associated with changing employment.

${ }^{19}$ Burnside, Eichenbaum, and Rebelo (1995) emphasize the role of capital utilization, which they measure using electricity usage.

${ }^{20}$ The relationship between marginal and average $q$ is found by differentiating equation (20) with respect to $K$ to obtain $q(y) \equiv V_{K}(K, X)=v(y)-y v^{\prime}(y)$. Using the expression for $q(y)$ in equation (26) to solve for $v(y)$, we obtain $v(y)=\frac{H}{1-\gamma} y^{\gamma}+\frac{E}{1-\alpha_{P}} y^{\alpha_{p}}$. Since $H>0$, $0<\gamma<1, \alpha_{P}>1$ and $E<0$, we have that $v^{\prime}(y)>0$. Thus average $q$ is an increasing function of $y$.

${ }^{21}$ Caballero and Leahy (1996) also find that Tobin's $q$ is a sufficient statistic for the investment state variable using their specification of fixed costs.

${ }^{22}$ Studies using Tobin's $q$ as an explanator of investment incorporating fixed costs include Abel and Eberly (1996a) and Eberly (1997). Alternatively, Caballero, Engel, and Haltiwanger (1995) estimate "mandated investment" as a measure of $y$ using plant data. 